\title{
Norois
}

Environnement, aménagement, société

\section{Progression de la Spartine (Spartina alterniflora Loisel) en rade de Brest (Finistère) entre 1952 et 2004 : de la mesure à la réponse gestionnaire} Expansion of Smooth Cordgrass (Spartina alterniflora) in rade of Brest

(Finistère) between 1952 and 2004: measurement and managing response

\section{Lenaïg Sparfel, Bernard Fichaut et Serge Suanez}

\section{(2) OpenEdition Journals}



Ce document a été généré automatiquement le 14 janvier 2022.

(c) Tous droits réservés 


\title{
Progression de la Spartine (Spartina alterniflora Loisel) en rade de Brest (Finistère) entre 1952 et 2004 : de la mesure à la réponse gestionnaire
}

\author{
Expansion of Smooth Cordgrass (Spartina alterniflora) in rade of Brest \\ (Finistère) between 1952 and 2004: measurement and managing response
}

Lenaïg Sparfel, Bernard Fichaut et Serge Suanez

\section{NOTE DE L'ÉDITEUR}

Cet article a été reçu le 14 avril 2005 et définitivement accepté le 22 septembre 2005.

Cette étude a été réalisée à la demande de la Direction de la Protection du Patrimoine Naturel et Sensible (DPPNS) de Brest Métropole Océane (BMO) ${ }^{1}$. Elle s'inscrit dans les orientations majeures du programme d'action du Contrat de Baie de la rade de Brest, l'objectif étant d'étudier la dynamique spatiale d'une Spartine américaine (Spartina alterniflora). Cette problématique découle des observations menées par le Conservatoire Botanique National de Brest, qui a montré que la progression de cette Spartine se faisait au détriment du schorre "originel ", menaçant ainsi de disparition une espèce végétale endémique à la rade, le Limonium humile (Miller), également nommé "petit statice " (Tesson et al., 1997 ; Quere et Magnanon, 1998 ; Quere et Magnanon, 2000). Face à ce constat, la DPPNS a lancé une étude de la dynamique spatiale de la Spartine en privilégiant l'approche quantitative, avec pour finalité, la proposition d'interventions permettant d'enrayer le développement de la plante (Sparfel, 2004 ; Fichaut et al., 2004). Dans un premier temps, le travail a consisté à réaliser une cartographie dynamique de la progression de la Spartine dans quelques rias et marais représentatifs de la rade de Brest, sur une période de 50 ans. La méthode utilisée repose sur l'analyse diachronique par photo-interprétation couplée à de la mesure de terrain au DGPS pour l'année 2004. 
Ces résultats ont permis de caractériser les modalités de son expansion. Dans un deuxième temps, nous avons cherché à mettre en évidence d'éventuels facteurs limitants expliquant sa répartition altitudinale. Enfin nous avons tenté de définir des méthodes opérationnelles visant à freiner localement la progression de la graminée.

\section{État de la question}

2 Spartina est une graminée vivace appartenant à la famille des Poacées. Le genre Spartina compte 14 espèces (Hubbard, 1992). Près de la moitié sont présentes en Europe dont une seule, Spartina maritima, est autochtone. Les 3 autres sont Spartina alterniflora introduite il y a plus de 150 ans en Angleterre, et Spartina townsendii (Groves) et Spartina anglica (C. E. Hubbard) qui toutes deux proviennent de l'hybridation des deux précédentes (Marchant, 1968a et b). Spartina alterniflora, originaire de la côte atlantique du continent nord-américain, s'est implantée accidentellement en rade de Brest, probablement à la fin de la Première Guerre mondiale, avec l'installation des bases américaines (Dizerbo, communication orale dans Bonnot-Courtois et al., 1989). Spartina anglica est aussi présente dans d'autres secteurs du littoral mer du Nord-MancheAtlantique, depuis le Bassin d'Arcachon jusqu'au Danemark.

3 Spartina alterniflora est une espèce native de la côte est des États-Unis où elle est dominante. Partout ailleurs, c'est une espèce introduite, y compris sur la côte ouest des États-Unis. Elle est rare et très localisée en Europe, sauf dans la rade de Brest où elle a colonisé plusieurs sites. Son expansion se fait à la fois en direction des schorres où les cortèges végétaux déjà installés sont, par voie de conséquence, supplantés par l'espèce, et sur les hautes slikkes nues qui se transforment progressivement en prairies monospécifiques. Dans ce cas, les aires de nourrissage des limicoles et autres oiseaux d'estrans voient leur superficie se réduire (Daehler et Strong, 1996).

4 Ce caractère envahissant a été observé à plusieurs reprises sans pour autant faire l'objet d'études quantitatives précises. Ainsi, les travaux menés en baie de San Francisco ont montré qu'entre le milieu des années 1970 et 1990, la colonisation de la slikke et des chenaux de marée a été très importante, la biomasse mesurée en 1989 était de $43 \mathrm{t}$ /ha (Callaway et Josselyn, 1992). En Baie de Willapa, située sur la côte ouest des États-Unis (état de Washington), l'augmentation des surfaces de Spartina alterniflora a atteint 60 \% entre 1994 et 1997 (Reeves, 1999 dans Grevstad et al., 2003). Pour le même secteur, les mesures effectuées par photo-interprétation entre 1970 et 1990 indiquent un accroissement concentrique des taches de l'ordre de $80 \mathrm{~cm} /$ an (Feist et Simenstad, 2000).

5 Quelques données existent également sur la dynamique spatiale de Spartina anglica, bien que cette dernière n'ait pas la même éthologie que Spartina alterniflora. C. H. Chung (1983) a montré qu'en Chine, les surfaces de Spartina anglica étaient passées de 110 ha en 1966 à 30000 ha en 1983, soit un taux de croissance annuel éqivalent de $39 \%$ par an. En baie de Kernic (Finistère Nord), les mesures effectuées sur la croissance des taches de Spartina anglica entre 1966 et 1988 montrent une augmentation concentrique de 15 $\mathrm{cm} / \mathrm{an}$, entraînant une colonisation presque totale des marges sud-ouest et orientale de la baie (Guénégou et al., 1991). Dans ce secteur, la Spartine qui apparaît en 1967, recouvrait $49000 \mathrm{~m}^{2}$ en 1989, soit un taux de croissance annuel équivalent de $60 \%$. 
6 Spartina alterniflora produit des semences viables mais se multiplie aussi végétativement par boutures des rhizomes. Généralement son extension se fait à partir de "taches circulaires individualisées » qui, par coalescence, finissent par constituer des prairies monospécifiques aux contours très arrondis. Cette morphologie typiquement révélatrice d'une organisation clonale, la différencie nettement des autres formations végétales de la zone intertidale, et a été particulièrement utile dans l'analyse photointerprétative.

7 Cette espèce présente une grande tolérance vis-à-vis de la plupart des facteurs écologiques. Concernant son amplitude altitudinale, la plupart des études mentionnent l'espace intertidal, sans plus de précision. T. Reidenbaugh (1983) et M. Gross et al., (1986) la situent entre le niveau moyen des basses mers et celui des hautes mers de vives-eaux. T. McKee et W. Patrick (1988), après étude bibliographique des articles traitant de son extension dans 33 marais de la côte est des États-Unis, avancent des données plus précises. Les auteurs montrent que dans un contexte micro à mésotidal, avec des marnages compris entre $0,39 \mathrm{~m}$ et $2,88 \mathrm{~m}$, elle est présente sur un gradient altitudinal légèrement inférieur à celui de la marée. La limite haute située au niveau des pleines mers moyennes en domaine mésotidal se rapproche de celui des hautes mers de vives eaux lorsque le marnage diminue. La limite basse se situe entre le niveau de la mi-marée et celui des plus basses mers. En baie de Fundy, où le marnage moyen est de $12 \mathrm{~m}$, la Spartine est présente à $2 \mathrm{~m}$ sous le niveau des pleines mers moyennes. Elle est recouverte à toutes les marées et peut rester immergée 3 heures par des hauteurs d'eau qui peuvent atteindre 3 mètres (Cranford et al., 1989).

8 Les données relatives au substrat sont généralement très vagues et évoquent simplement la slikke (mudflats). Toutefois, S. Broome et al., (1986) indiquent la présence de prairies de Spartines sur des substrats sableux contenant 0,5\% de limons et argiles. Cette macrophyte peut donc potentiellement coloniser tous les types de slikkes. En baie du Kernic, l'hybride Spartina anglica est signalée sur des sables fins envasés ( $20 \%$ de limons et argiles) - (Guénégou et al., 1991).

9 L'hydrodynamisme apparait être un facteur limitant pour toutes les espèces des marais. Toutes choses égales par ailleurs, la limite inférieure atteinte par les Spartines s'abaisse lorsque la longueur du fetch des houles diminue (Swales et al., 2004).

10 Dans la nature, l'espèce semble s'accommoder de salinités variables. S. Broome et al., (1986) citent des valeurs comprises entre 20 et $35 \%$. Les observations menées in situ ou expérimentalement sous serre montrent que dans les milieux peu salés, ou occasionnellement dessalés par des apports d'eau douce, la production et la biomasse tendent à augmenter fortement (Linthurst et Seneca, 1981 ; Broome et al., 1986).

\section{Choix des sites et méthodologie}

11 Trois secteurs représentatifs de l'ensemble des marais maritimes de la rade de Brest ont été retenus (fig. 1). Les secteurs de Pont-Callec sur la commune de PlougastelDaoulas, du Pédel sur les communes de Plougastel-Daoulas et de Loperhet, et de Mengleuz sur les communes de Daoulas et de Logonna-Daoulas. Ces différents sites présentent les trois morphologies caractéristiques de l'ensemble des zones humides de la rade de Brest (Guilcher et al., 1982). 


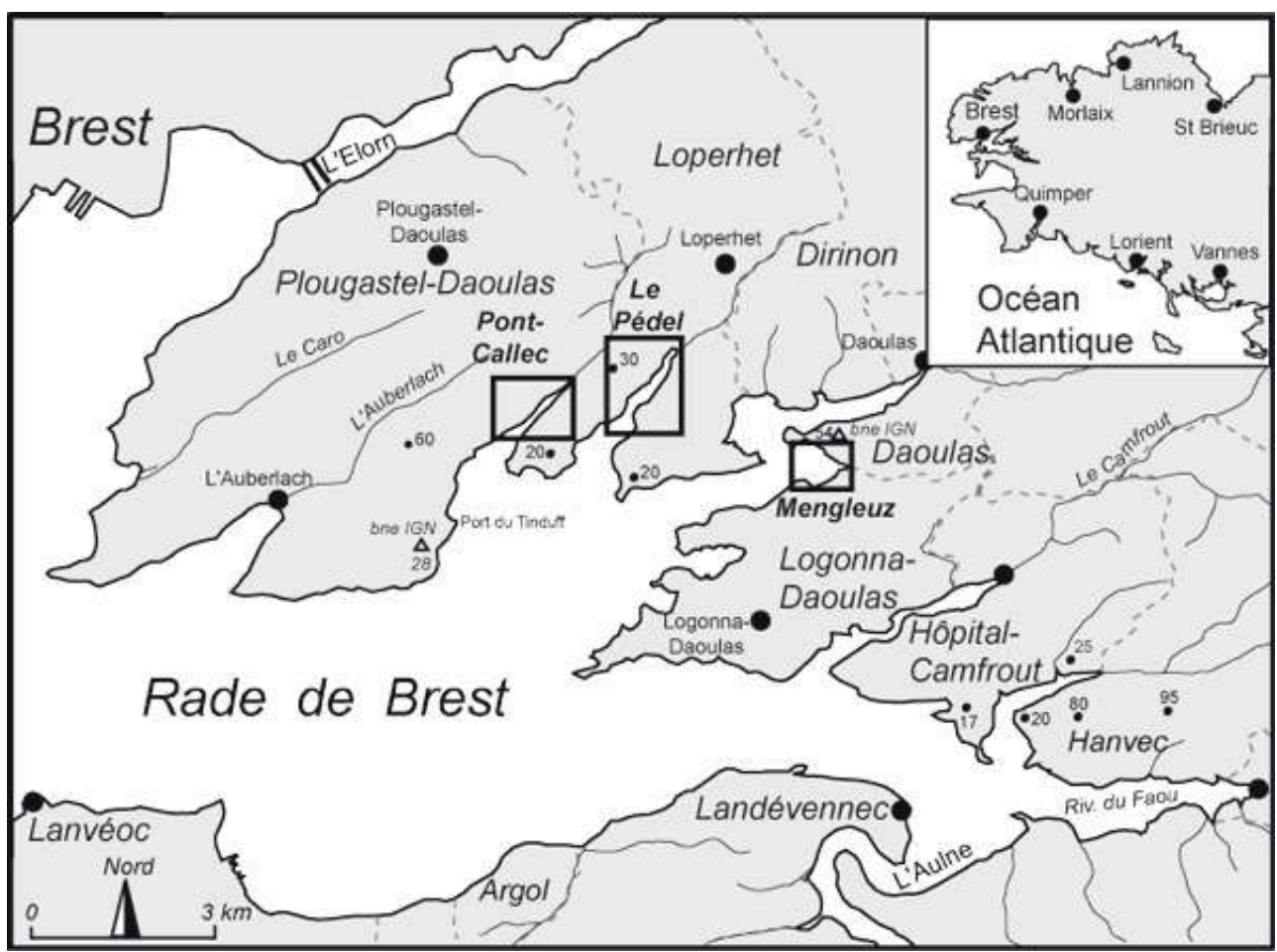

- Le site de Mengleuz correspond à un marais salé qui s'est développé en arrière de deux cordons littoraux en chicane. Sa superficie atteint $87000 \mathrm{~m} 2$. En dehors des abords immédiats de l'unique chenal médian, il est entièrement constitué de haute slikke et de schorre végétalisés. Du fait du colmatage important, les chenaux secondaires sont rares et dessinent un réseau de type fasciculé (Verger, 2005). La pente longitudinale générale du chenal médian est de $7 \%$ alors que celle des bancs latéraux de slikke et de schorre est comprise entre 0,7 et $2,7 \%$. En dehors du lit du chenal principal qui est tapissé d'un mélange de cailloux et graviers, le matériel de colmatage est exclusivement vaseux.

Les secteurs de Pont-Callec et du Pédel correspondent à d'anciennes vallées ennoyées appelées rias, colmatées par du matériel fluvio-marin.

- Sur le site de Pont-Callec, les Spartines sont cantonnées dans la partie amont et c'est ce secteur seul qui a été étudié. Cette zone étroite, large au maximum de 150 mètres couvre une superficie d'environ $110000 \mathrm{~m} 2$. La pente longitudinale générale du chenal médian est là encore de $7 \%$, par contre les vasières situées de part et d'autre ont une pente bien plus marquée qu'à Mengleuz, avec des valeurs comprises entre 2,5 et $10 \%$. Le matériel déposé dans le chenal principal passe de caillouteux en amont, à sablo-vaseux vers l'aval. La partie basse des slikkes est extrêmement vaseuse (entre 80 et $90 \%$ ) de pélites. Vers le haut, la part des fines tend à diminuer au profit d'une proportion plus importante de sables et de graviers (de 70 à $95 \%$ )

- Sur le site du Pédel, pour les mêmes raisons qu'à Pont-Callec, seule la partie amont de l'estuaire a été étudiée. Cet ensemble de $130000 \mathrm{~m}^{2}$ est large au maximum de $250 \mathrm{~m}$. Comme à Pont-Callec, le chenal principal ne reçoit que quelques affluents secondaires situés dans le prolongement de petis ruisseaux continentaux. Si la pente longitudinale du chenal médian est plus forte qu'à Pont-Callec (11\%), ce dernier est toutefois moins encaissé. Comme en plus la ria est plus large, les pentes des vasières latérales sont moins importantes et comprises 
entre 2,5 et $6 \%$. Le fond du chenal, caillouteux en amont est alternativement sableux et vaseux ensuite. Les slikkes et schorres sont constitués de vases contenant 30 à $70 \%$ de pélites. Tout à fait à l'aval, le haut d'estran est gravelo-sableux.

L'étude diachronique de la dynamique spatiale de la Spartine a été réalisée par photointerprétation numérique couplée à de la mesure de terrain. L'ensemble de ces données a été traité sous Système d'Information Géographique (SIG) à l'aide du logiciel Mapinfo. Ainsi, des photographies aériennes IGN couvrant la période allant de 1952 à 2000 avec une fréquence de prise de vues de 8 à 10 ans, ont été utilisées pour chaque site (fig. 2). La méthodologie retenue pour la photo-interprétation numérique est la suivante :

- dans un premier temps, les photographies aériennes ont été numérisées à très haute résolution de manière à obtenir un pixel proche de $50 \mathrm{~cm}$ sur le terrain (cette résolution correspond à celle des ortho-photographies IGN de 2000 ayant été utilisées comme image de référence pour la correction géométrique);

- dans un second temps, ces photographies ont été géométriquement corrigées afin d'éliminer les phénomènes de déformation inhérents à la prise de vue. Nous avons utilisé pour cela la méthode de saisie des points d'amer en prenant pour document de référence l'orthophotographie aérienne IGN de 2000. Des marges d'erreur ont été calculées pour chaque secteur afin de tester la qualité des corrections géométriques. Les décalages d'une image à une autre après correction sont inférieurs à +/-3 m ;

- l'acquisition des états de surface végétalisée s'est faite sous le logiciel de SIG (MapInfo). Il a fallu tout d'abord géoréférencer l'ensemble des données images, les contours des formations végétales ont été ensuite numérisés à l'écran. La typologie des états de surface reprend les diverses formations végétales du schorre et de la haute-slikke (cf. légendes des figures 3, 5 et 6) ;

- Enfin, l'évolution des surfaces de Spartine et de schorre a donné lieu à une cartographie dynamique à partir de laquelle le calcul des surfaces et des vitesses de progression de la Spartine a été effectué pour chaque date.

La cartographie réalisée en 2004 repose sur des relevés de terrain. Ils ont été effectués à l'aide d'un DGPS' (Differential Global Positional System). Les mesures ont été effectuées en mode RTK (Real Time Kinematics) avec une précision horizontale et verticale respective de $2 \mathrm{~cm}(\mathrm{x}, \mathrm{y})$ et de $1,5 \mathrm{~cm}(\mathrm{z})$. Les mesures ont été raccordées au système géodésique français à partir de deux bornes IGN ${ }^{3}$ situées pour la première à $250 \mathrm{~m}$ au nord du site de Mengleuz, au niveau du hameau de Rosmélec, la seconde se trouvant au sud du port $\mathrm{du}$ Tinduff, sur la commune de Plougastel-Daoulas (fig. 1). Le système de géoréférencement qui a été retenu est le Lambert II étendu.

Enfin, l'analyse portant sur l'amplitude altitudinale de la Spartine a été également réalisée à partir de levés de profils topographiques au DGPS. Ils ont été positionnés transversalement à l'axe des rias ou des chenaux principaux, de manière à mettre en évidence l'étagement de la végétation (cf. fig. 7, 8 et 9). 
Figure 2 : Inventaire des photographies aériennes IGN du site de Mengleuz utilisées pour l'étude diachronique

Inventory of the IGN aerial photographies of Mengleuz area used for diachronic analysis

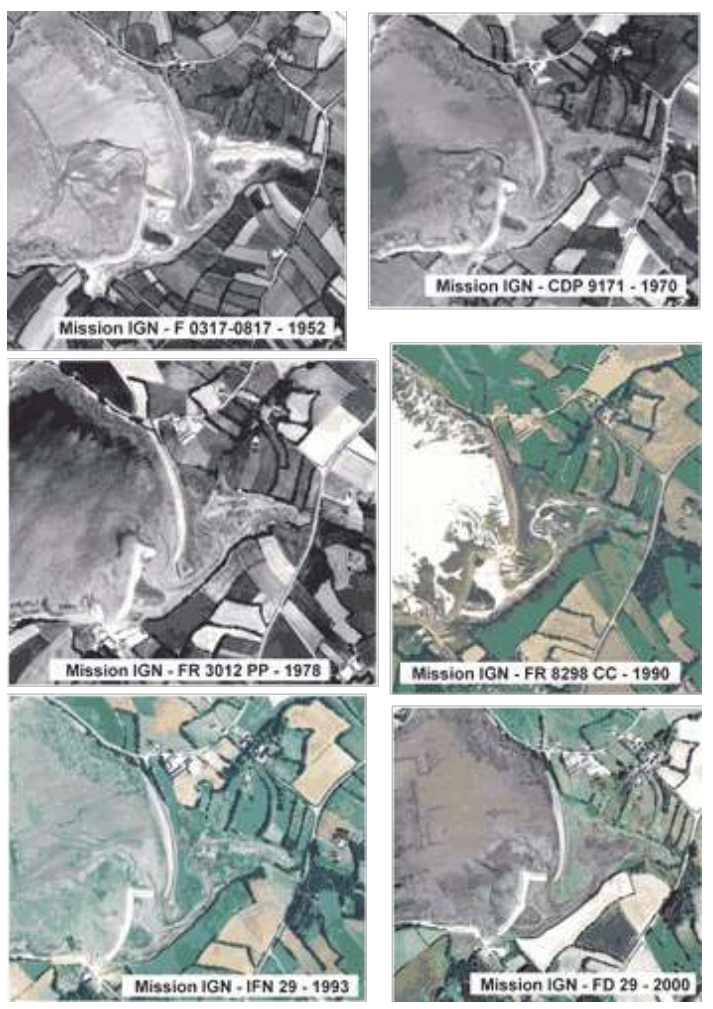

\section{Progression très rapide de la Spartine}

Les résultats obtenus sur les trois sites montrent que la progression de la Spartine a été très rapide entre 1952 et le milieu des années 1990. À partir de cette date, la diminution des espaces colonisables a induit une baisse importante des vitesses de progression. 
Figure 3 : Cartographie diachronique dynamique de la progression de la Spartine entre 1952 et 2004 pour le site de Mengleuz

Diachronic mapping of the progression of Spartine between 1952 and 2004 in Mengleuz site

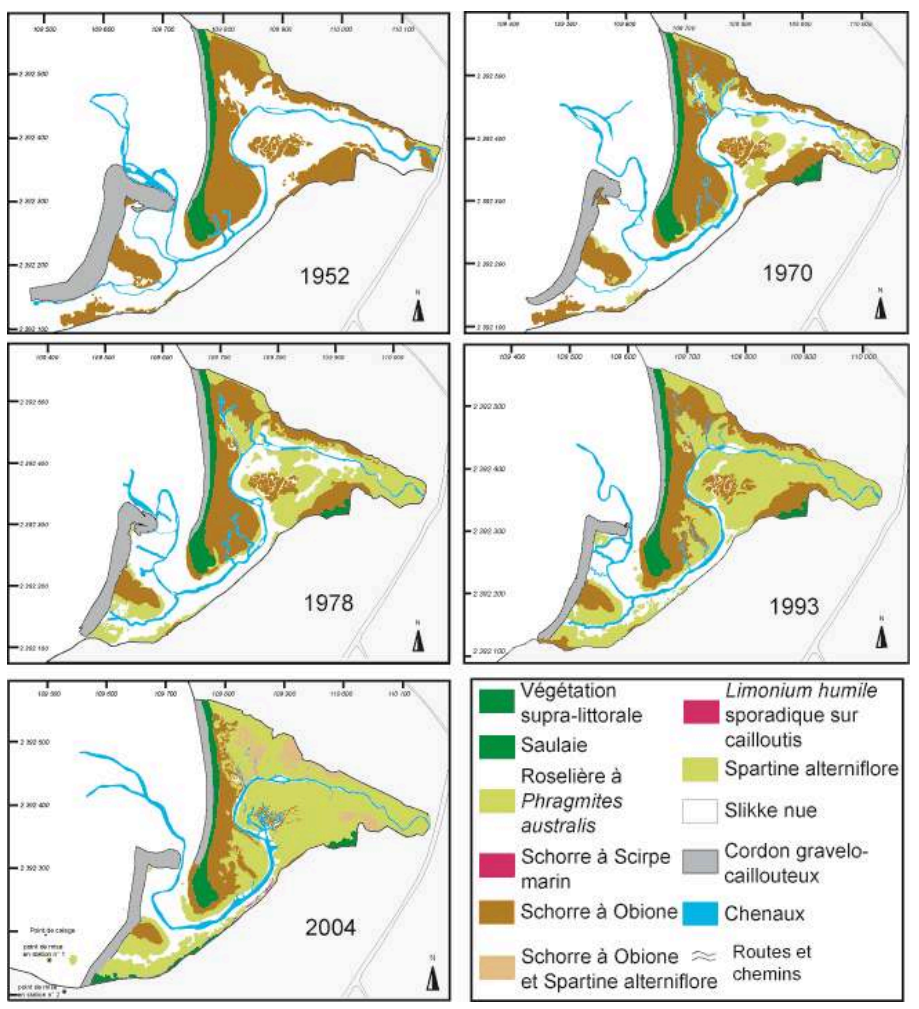

17 Le caractère abrité du site de Mengleuz explique qu'en 1952 le schorre soit déjà bien développé. Il couvre une surface d'environ $32930 \mathrm{~m}^{2}$, pour une superficie totale du marais avoisinant $84000 \mathrm{~m}^{2}$ (fig. 4). À cette époque, la Spartine était absente. La date de son implantation est inconnue, mais en 1970 elle couvre $9400 \mathrm{~m}^{2}$. À partir de cette date, l'envahissement du marais par la graminée est continu. En l'espace de 34 ans, la superficie colonisée par la plante a presque été multipliée par quatre, avec un taux de croissance annuel équivalent de $4 \%$ entre 1970 et 2004. 
Figure 4 : Quantification de la progression de la Spartine (surface et vitesse) sur les 3 sites d'étude Quantification of spreading of Spartina (surface and speed) on the 3 study sites

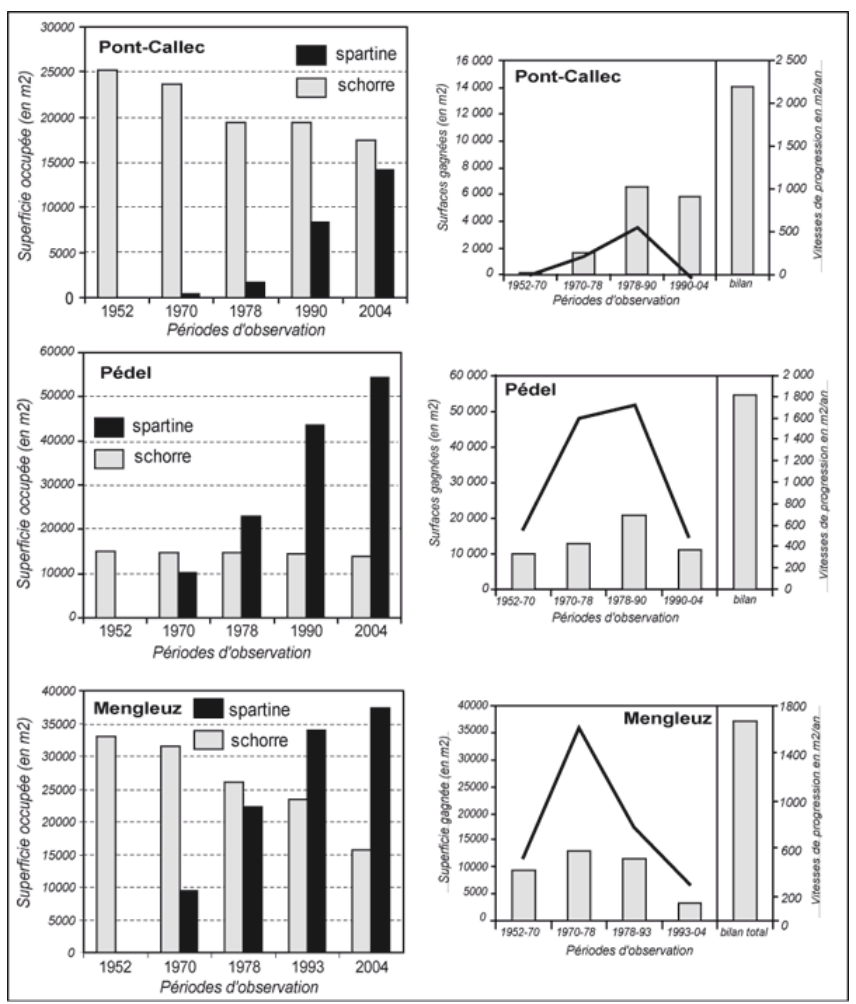

progression s'effectue dans toutes les directions, elle répond toutefois à un certain rythme morpho-chronologique. Dans un premier temps, la Spartine s'est implantée dans la quasi-totalité de la haute slikke. Les vitesses de colonisation sont alors les plus importantes avec un accroissement moyen de $1600 \mathrm{~m}^{2}$ par an durant la période 1970-1978. Ensuite, la colonisation s'est faite jusqu'aux abords immédiat du chenal médian et au sein même du schorre initial. Au cours de cette seconde étape, la progression a été plus lente mais s'est accompagnée du colmatage d'une grande partie des chenaux secondaires (fig. 3 et 4). 
Figure 5 : Cartographie diachronique dynamique de la progression de la Spartine entre 1952 et 2004 pour le site de Pont-Callec

Diachronic mapping of the progression of Spartine between 1952 and 2004 in Pont-Callec site
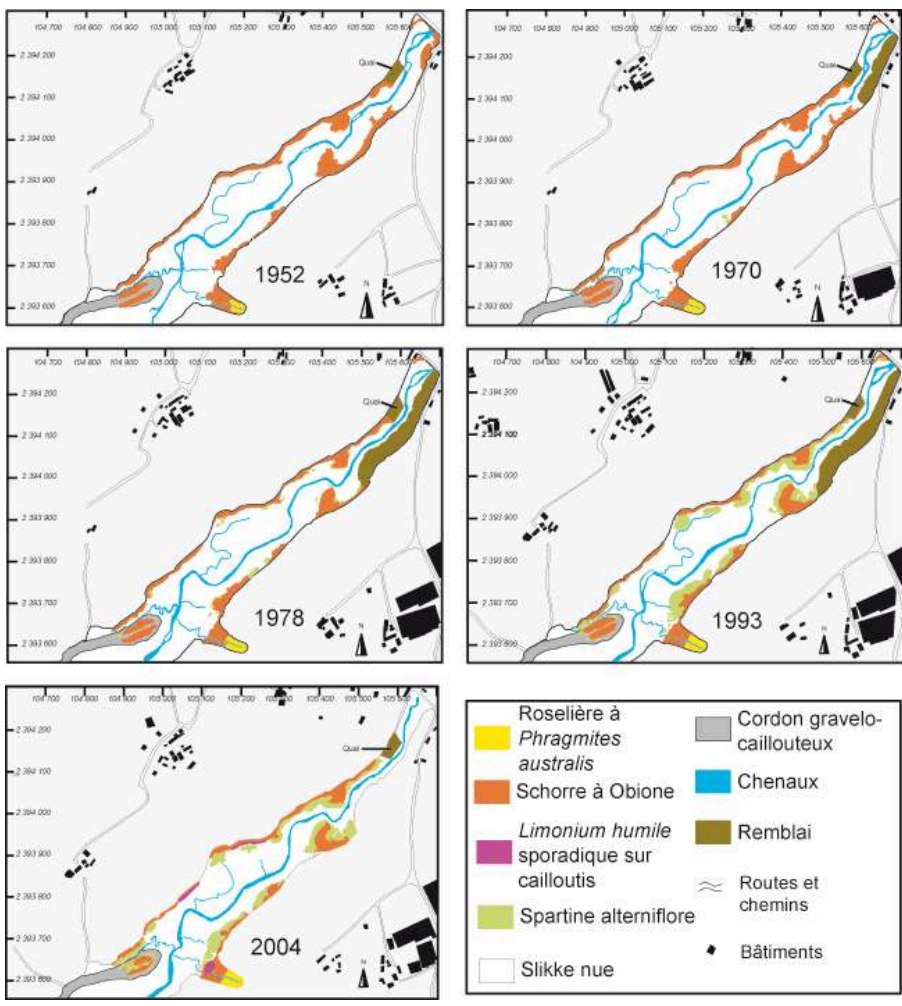

En 1952, le site est peu végétalisé et se limite à un schorre frangeant de $25140 \mathrm{~m}^{2}$ pour une surface totale de la zone humide de $118500 \mathrm{~m}^{2}$ (fig. 4).

En 2004, la superficie du schorre n'est plus que de $17350 \mathrm{~m}^{2}$. Cette régression découle en grande partie du remblaiement de la rive gauche à l'amont de la ria, et secondairement d'une expansion de la Spartine aux dépens des marges inférieures du schorre. Actuellement elle recouvre $14000 \mathrm{~m}^{2}$, soit $11 \%$ de la zone étudiée. La graminée est apparue au début des années 1970, sous forme de taches de superficie extrêmement réduite sur la partie haute de la slikke en rive gauche. Sa vitesse de progression a été croissante entre 1970 et 1993, passant de $250 \mathrm{~m}^{2}$ par an entre 1970 et 1978, à $2000 \mathrm{~m}^{2}$ par an entre 1990 et 1993 (fig. 4). À partir de cette date la superficie colonisée reste stable. Le taux d'expansion annuel équivalent enregistré pour la période allant de 1970 à 1993 est de $24 \%$. 
Figure 6 : Cartographie diachronique dynamique de la progression de la Spartine entre 1952 et 2004 pour le site du Pédel

Diachronic mapping of the progression of Spartine between 1952 and 2004 in Pédel site
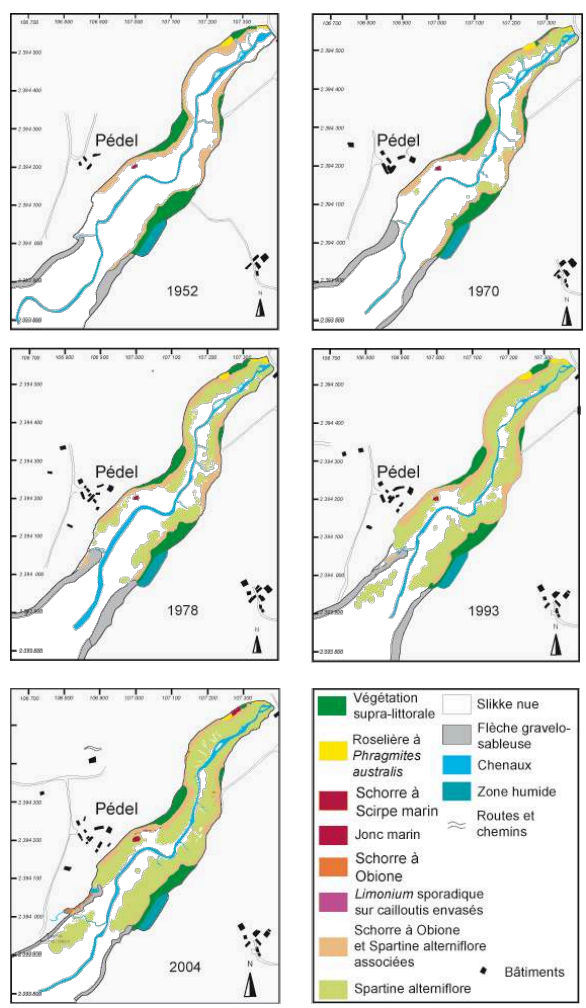

En 1952, un peu comme à Pont-Callec, les surfaces végétalisées sont cantonnées aux marges de la ria. Le schorre couvre $15000 \mathrm{~m}^{2}$ des $118000 \mathrm{~m}^{2}$ de la zone d'étude. En 1970, la Spartine est déjà bien installée et occupe $10150 \mathrm{~m}^{2}$ au fond de la ria, dans la partie supérieure des slikkes au contact du schorre. Par la suite, la progression s'est faite de deux manières. En amont, les prairies déjà installées se sont étendues graduellement vers le chenal médian. Cette colonisation a favorisé un exhaussement graduel de la slikke qui aujourd'hui forme la haute slikke se terminant en bordure de chenal par un talus marqué dont la hauteur peut dépasser un mètre (fig. 9). Vers l'aval, l'extension s'est d'abord faite par taches initialement individualisées dans la partie supérieure des vasières. Ensuite, par coalescence, ces taches ont fini par former de vastes étendues monospécifiques. La progression la plus importante s'est effectuée entre 1970 et 1993, avec des vitesses de progression allant de $1600 \mathrm{~m}^{2}$ par an pour la période 1970-1978 à $1720 \mathrm{~m}^{2}$ par an pour la période 1990-1993. Aujourd'hui, la Spartine recouvre une surface de $54400 \mathrm{~m}^{2}$, soit $46 \%$ de la ria (fig. 4), mais l'extension vers l'aval se fait beaucoup plus lentement. Le taux de croissance annuel équivalent enregistré pour la période allant de 1970 à 1993 est de $5 \%$.

\section{Modalités de progression de la Spartine}

La progression de la Spartine s'est effectuée de manière différente pour chacun des sites. En effet, la dynamique spatiale de la graminée sur Mengleuz et au Pédel semble avoir été sensiblement la même. Dans un premier temps, elle s'est installée en amont du site, sur des vases dessalées par des arrivées d'eau douce, et dans la partie haute de la slikke nue. Ensuite, à partir de ces bastions initiaux, elle a graduellement progressé 
vers le chenal principal au fur et à mesure de l'exhaussement du substrat. Dans le même temps, de nouvelles zones de colonisation sont apparues vers l'aval au milieu des vasières des deux rives. Puis, de là, l'expansion s'est faite dans toutes les directions y compris au sein même des schorres déjà présents, peu à peu colonisés à partir de la haute slikke et des chenaux secondaires progressivement envahis, tronçonnés et éventuellement totalement colmatés. Aujourd'hui, les deux sites sont très largement recouverts par la Spartine, et la progression de cette dernière ralentit pour des raisons que nous aborderons dans le paragraphe suivant.

Le site de Pont-Callec est à mettre à part. Même si c'est une ria comme au Pédel, l'extension de la Spartine ne s'est pas réalisée de façon similaire. Les premières touffes de la graminée invasive sont apparues non pas au fond de la ria cette fois, mais vers l'aval, sans doute juste avant 1970. De 1970 à 1978 d'autres taches se sont installées vers l'amont pour finalement atteindre la cale. C'est après 1978 seulement que la jonction des touffes entre elles a abouti à la formation de prairies. Toutefois, même si l'espace qu'occupe cette plante s'est étendu, la graminée est loin de recouvrir aujourd'hui la même superficie que sur les deux autres sites. De plus, elle s'est cantonnée à la haute slikke en bordure du schorre à obione, sans s'approcher du chenal, pour des raisons qui sont développées ci-dessous.

\section{Amplitude altitudinale et facteurs écologiques limitants}

Nous avons tenté de voir s'il y avait une correspondance entre l'altitude du substrat et l'installation de la Spartine, c'est-à-dire de définir l'espace qu'elle peut potentiellement coloniser. Pour ce faire, une analyse fine de la répartition de l'espèce a été menée sur les profils topographiques réalisés sur chacun des sites (fig. 7, 8 et 9).

Figure 7 : Étagement des formations végétales sur le site de Mengleuz Altitudinal repartition of plant associations in Mengleuz site

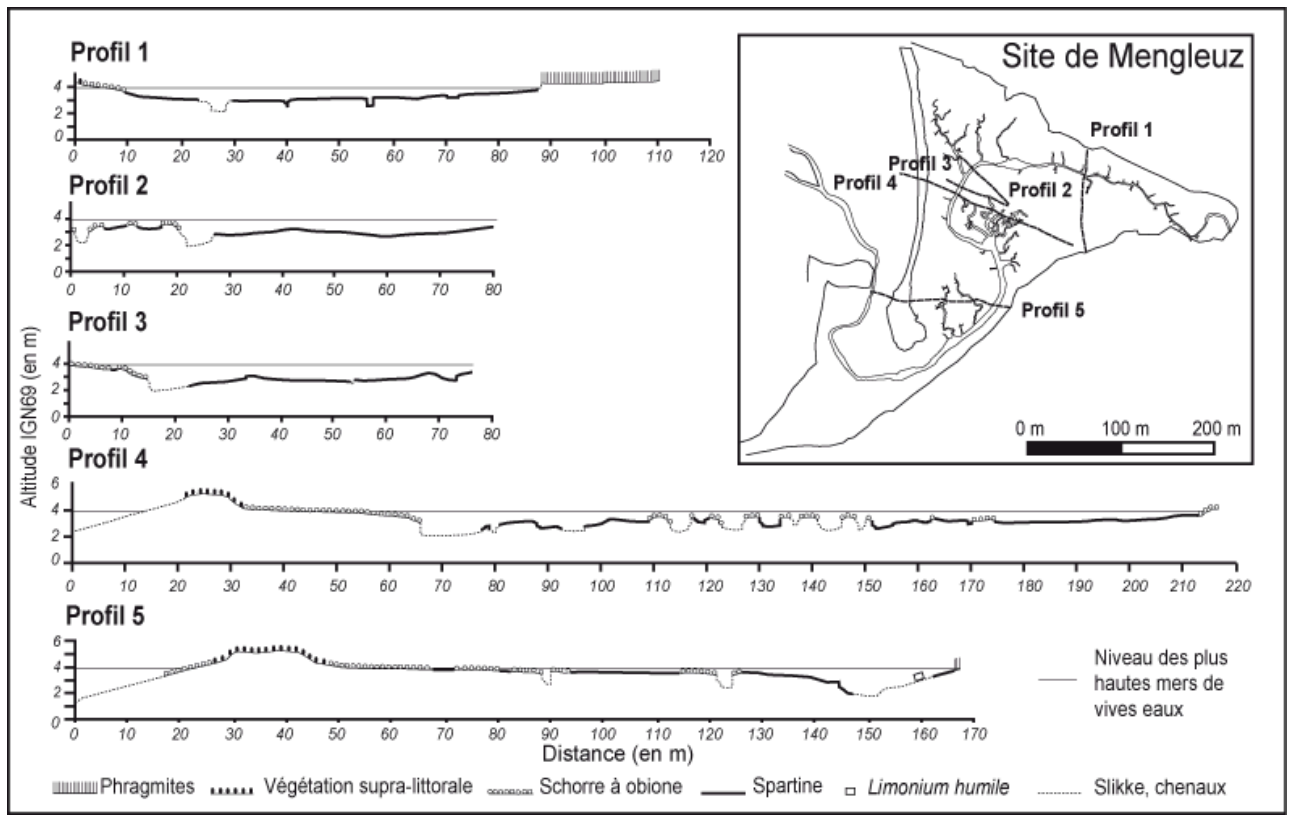


En rade de Brest, dans un contexte macrotidal, le marnage atteint 7,32 $\mathrm{m}$. Le niveau moyen de la mer correspondant au zéro IGN69 situé à 4,02 $\mathrm{m}$ au-dessus du zéro hydrographique est utilisé ici comme référent altitudinal. Dans tous les sites étudiés l'amplitude altitudinale de la Spartine apparaît nettement décalée, par rapport à ce qu'indique la bibliographie.

Figure 8 : Étagement des formations végétales sur le site de Pont-Callec Altitudinal repartition of plant associations in Pont-Callec site

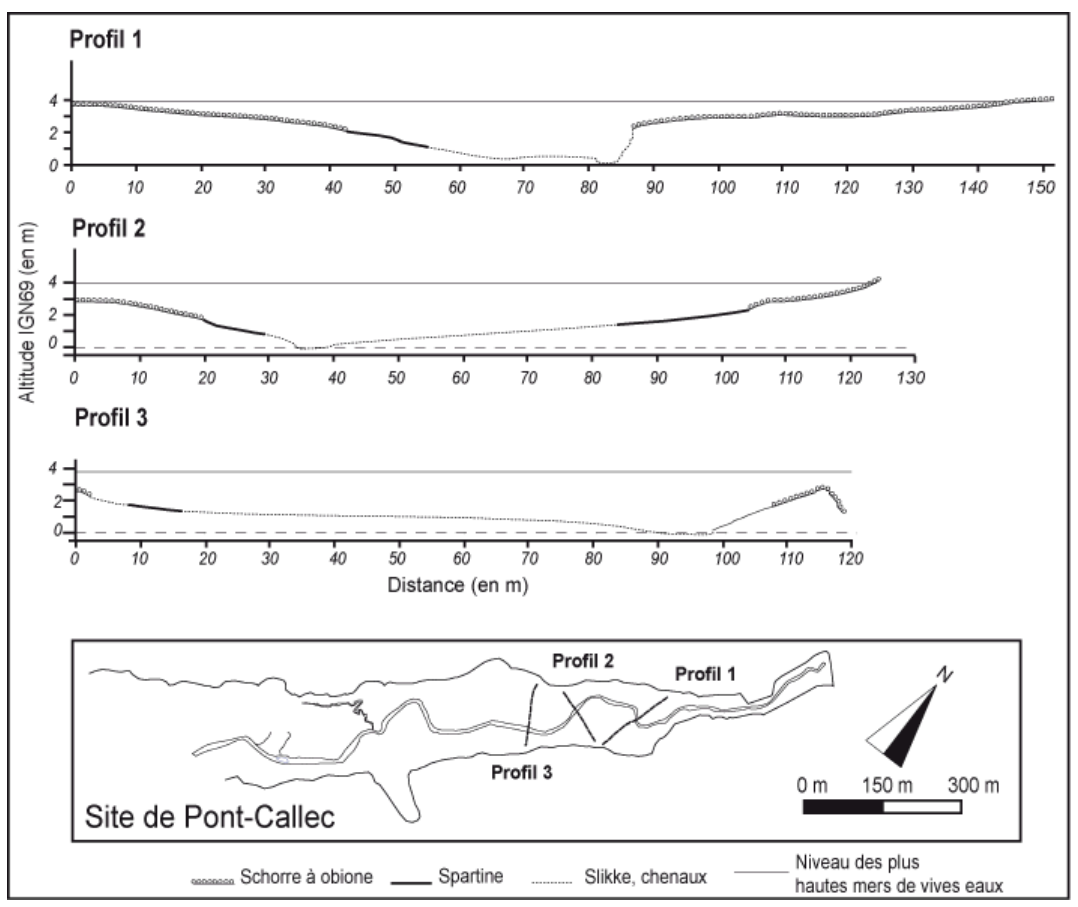

Vers le haut, l'espèce atteint partout le niveau des plus hautes mers d'équinoxe et peut même le dépasser (3,8 m à Mengleuz au-dessus du zéro IGN69). Elle est même présente en périphérie du marais, dans les ourlets de phragmites installés sur des vases dessalées par des arrivées d'eau douce continentale. Le fait qu'elle envahisse peu à peu les schorres en dépit de la compétition interspécifique (fig. 3) montre que, vers les hauts niveaux, aucun facteur ne semble en mesure d'enrayer son installation.

La limite basse est comprise dans une fourchette altitudinale extrêmement réduite ( 0,9 à $1,4 \mathrm{~m}$ ) correspondant au niveau des pleines mers de mortes-eaux de coefficient 30 à 40. Cette limite correspond à peu près à celle qu'indiquent J. Levasseur et M.-C. Guenegou (1988) pour Spartina anglica sur la côte nord du Finistère. À Mengleuz elle se situe même plus haut, entre 1,9 et $2,9 \mathrm{~m}$, ce qui s'explique par l'altitude élevée du marais dans lequel le fond du chenal médian est compris entre $2,2 \mathrm{~m}$ en amont de la ria et $1,7 \mathrm{~m}$ au niveau de l'exutoire. Ici la Spartine occupe tous les bancs de slikke latéraux, fortement exhaussés depuis son installation, qui dominent le chenal médian par un talus fortement marqué. Seule la présence permanente de l'eau fait alors obstacle à son installation dans le chenal. À Pédel, où l'altitude du chenal est comprise entre 0,2 et 0,9 $\mathrm{m}$, la limite inférieure des Spartines est partout comprise entre 1,1 et 1,2 $\mathrm{m}$. Si en amont l'espèce s'étend jusqu'au sommet du talus bordant le chenal, vers l'aval elle ne parvient pas à coloniser la partie basse des bancs de slikke nue dont la pente est extrêmement faible (profil 4, fig. 7). Pourtant elle est présente sur la partie haute de ces bancs depuis au moins 1970, et c'est même dans leur partie médiane que la propagation 
a été la plus rapide. En l'absence de compétition interspécifique, il est clair que les limites à son expansion vers le bas découlent de facteurs abiotiques. L'extrême régularité de l'altitude de la base des spartinaies ne semble pas être imputable à une diminution du drainage du substrat fréquemment évoquée (McKee et Patrick, 1988). En effet, elle pousse très bien et constitue même des peuplements monospécifiques dans des dépressions mal drainées à Mengleuz (profil 2, fig. 7). Le facteur limitant semble donc être directement lié à la longueur de la période de submersion.

Figure 9 : Étagement des formations végétales sur le site du Pédel Altitudinal repartition of plant associations in Pédel site

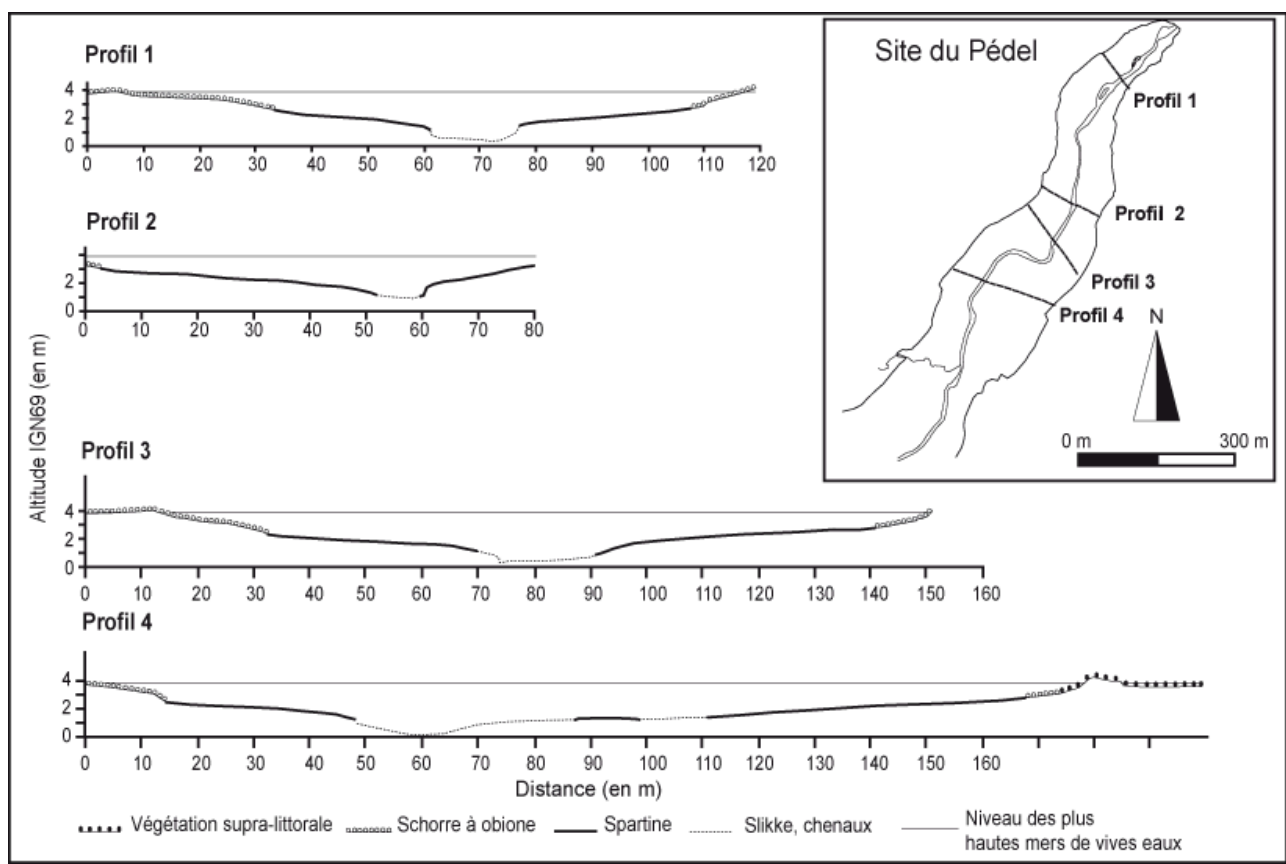

Les observations faites à Pont Callec semblent corroborer cette hypothèse. En effet, dans cette ria, la base de certaines taches est à $0,9 \mathrm{~m}$ seulement (fig. 8). Toutefois cette altitude est rarement atteinte et à ce niveau on note aussi une forte mortalité, attestée, en pleine saison végétative, par la présence de Spartines réduites à l'état de chaumes. Il semble donc qu'à cette altitude, l'installation soit possible mais pas durable.

En dehors de la durée de submersion qui, lorsqu'elle est trop longue, semble limiter la progression de l'espèce, un autre facteur, l'hydrodynamisme et ses implications sur la nature du matériel sédimentaire, paraît être l'autre facteur limitant. Au Pédel, immédiatement à l'est du hameau sur la rive concave d'un méandre du chenal principal, la Spartine ne parvient pas à s'installer. À cet endroit, directement exposé au fetch le plus long de la ria, le substrat est gravelo-caillouteux alors que partout autour il est vaseux ou vaso-sableux. L'absence de vase, liée à la forte agitation empêche l'installation de la Spartine et permet le maintien d'une frange de végétation abritant le Limonium humile. À Mengleuz, on trouve une situation comparable sur la rive gauche et concave du chenal principal, au droit de l'extrémité de la flèche interne du complexe en chicane. Là aussi le substrat est gravelo-caillouteux, mais dans ce cas c'est la compétence des courants de marée plaqués contre la berge qui explique l'absence de vase et le maintien du Limonium dans ce secteur. 
En conclusion, dans les marais maritimes de la rade, la Spartine peut coloniser tout l'espace littoral compris entre le niveau atteint par les plus hautes mers et celui des pleines mers de mortes eaux de coefficient 40 environ. Vers le haut, où elle peut remplacer toute végétation préexistante, rien ne semble en mesure d'empêcher son extension sans doute pour le moment inachevée. Vers le bas, seule la présence permanente d'eau dans les principaux chenaux ou la durée de submersion trop longue sous le niveau des pleines mers de mortes eaux, peut empêcher son installation. Très localement, lorsque l'hydrodynamisme empêche le dépôt de vase, la Spartine est absente à des niveaux qu'ailleurs elle colonise rapidement.

\section{Comment contrôler l'expansion de la Spartine en rade de Brest?}

31 Au terme de cette étude il nous a été demandé de proposer des solutions permettant de freiner, voire même de stopper la progression de la Spartine, dans le but de protéger le Limonium humile. Ce dernier est présent dans les rias et marais salés de la partie orientale de la rade qui sont tous, dans le même temps, en voie de colonisation par la Spartine (fig. 10) à l'image des trois sites traités dans le cadre de cette étude. De nombreuses techniques ont été mises en œuvre en France et à l'étranger. Le traitement par herbicide, généralement à base de glyphosate, est le plus répandu. Cette méthode a été utilisée dans le parc naturel de Northumberland en Grande-Bretagne (Garnett et al., 1992), mais également aux États-Unis et en Australie. Si elle s'avère efficace, elle présente un coût économique important et a des impacts écologiques qui restent à déterminer précisément (Davey, 1993). Les techniques visant à la destruction par fauchage (Daehler, 1996), ou par enfouissement, peuvent être très efficaces. Le labourage au rotolabour, suivi d'épandage de chaux a également été utilisé en baie de Somme (Sournia et al., 2000). Rappelons enfin les techniques reposant sur la lutte biologique par introduction d'insectes phytophages tels que Prokelisia marginata. Cette méthode vient d'être mise en œuvre dans la baie de Willapa (Washington) sur des prairies de Spartina alterniflora (Grevstad et al., 2003). 
Figure 10 : Répartition du Limonium humile (Miller) dans la partie orientale de la rade de Brest Repartition of Limonium humile in the eastern part of rade de Brest

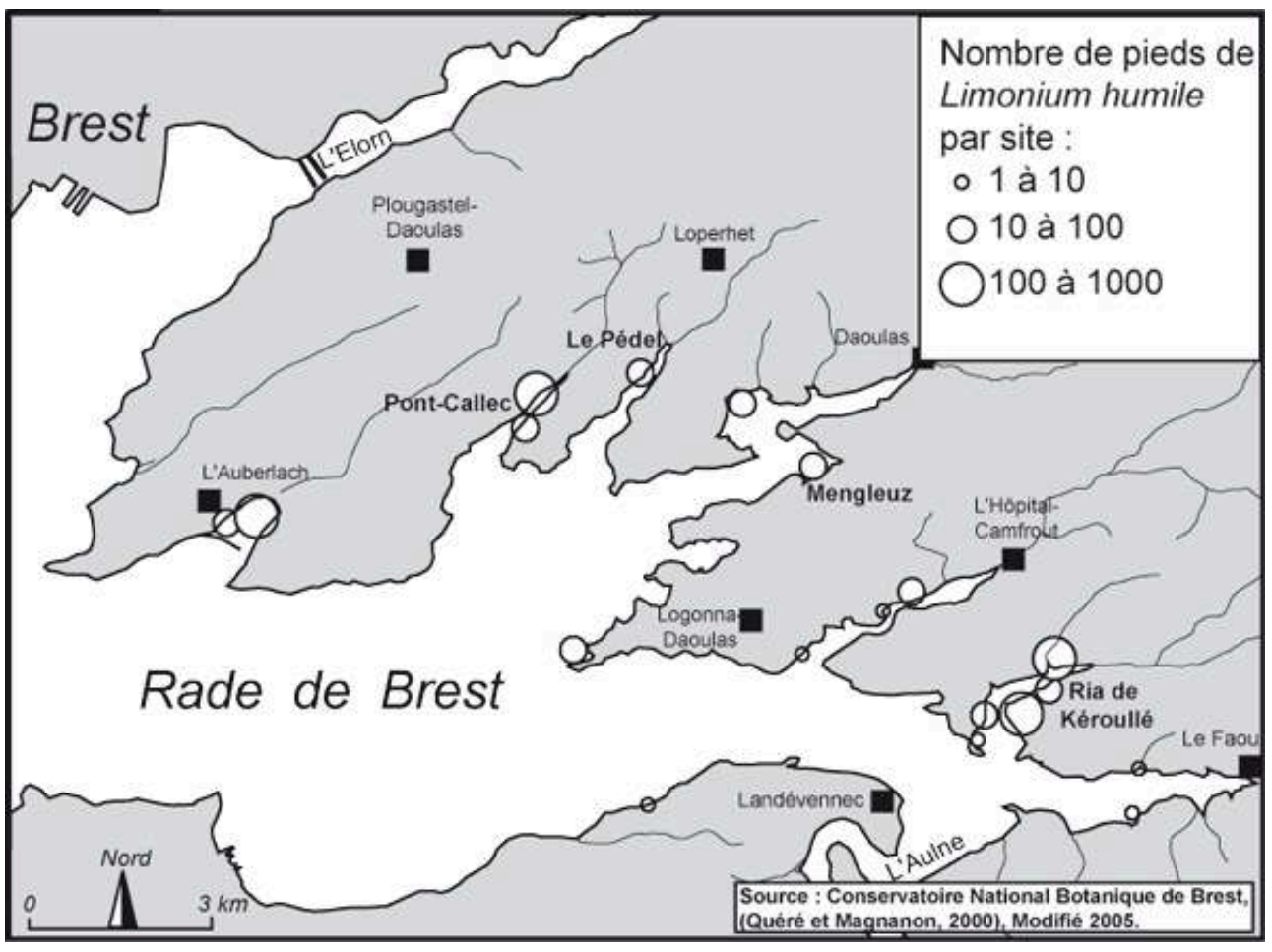

Quel que soit le type de traitement envisagé, toutes les études montrent qu'une fois la plante installée sur de grandes superficies, son éradication est impossible ou indésirable, puisqu'elle risquerait d'avoir des conséquences néfastes sur les autres composantes des milieux estuariens. Les méthodes mécaniques à grande échelle impliquent une circulation d'engins difficile à mettre en œuvre sur les vases de la rade de Brest. Quant à l'emploi d'herbicides, au-delà même de considérations relatives à la légalité de l'application des produits, s'avère impossible à partir du moment où le but de l'intervention se veut sélectif et vise à préserver au moins une espèce végétale. Localement, des solutions à grande échelle ou de type préventif peuvent toutefois donner des résultats. C. Daehler et D. Strong (1996) citent le cas du Puget Sound (côte ouest des États-Unis) où Spartina alterniflora ne parvient pas à se répandre, car dès leur apparition, les touffes sont systématiquement recouvertes de bâches de plastique noir et s'en trouvent étouffées. En rade de Brest, le Conservatoire Botanique National de Brest a testé cette méthode, ou celle qui consiste à entourer les secteurs colonisés par le Limonium humile de plaques de PVC profondément enfoncées dans la case, de manière à empêcher la propagation de la Spartine par extension des rhizomes (Quéré et Magnanon, 1998; Quere et Magnanon, 2000). Les résultats sont satisfaisants, mais exigent un suivi et des interventions réguliers et ne peuvent concerner que de petites superficies.

Parallèlement, notre étude montre que dans certains secteurs, l'hydrodynamisme, et par voie de conséquence la nature grossière du substrat, freinent l'implantation massive de la Spartine. Là, le Limonium parvient à se maintenir pour le moment. Toutefois les quelques touffes présentes sont appelées à s'étendre ne serait ce que parce qu'elles vont favoriser l'atterrissement de vase. En ces lieux, une mesure conservatoire simple consisterait à détruire systématiquement, éventuellement par arrachage, les quelques taches de Spartines présentes et à évacuer les poches de vase qui les 
supportent. Une première mesure de sauvetage d'urgence reviendrait à identifier tous les secteurs à Limonium où l'hydrodynamisme et d'autres facteurs limitants s'opposent à la colonisation inéluctable par la Spartine, et à y appliquer les mesures préconisées cidessus.

\section{Discussion}

34 À l'échelle de la rade de Brest, cette étude présente pour la première fois des données chiffrées sont produites sur la progression de la Spartine dans la rade de Brest depuis 50 ans. Des taux de progression annuels équivalents de $4 \%$ à $24 \%$ ont été enregistrés, ils restent bien inférieurs à ceux mesurés dans le cas de Spartina anglica, en baie de Kernic (60\%) - (Guénégou et al., 1991), ou en Chine (39\%) - (Chung, 1983). Ces différences peuvent s'expliquer par le fait qu'il s'agit d'espèces différentes, d'une part. D'autre part, les vasières de la rade de Brest, localisées sur des berges de rias, sont étroites et pentues, donc, des facteurs physiques limitants s'exercent efficacement pour freiner l'expansion de l'espèce. De plus l'analyse de la répartition altitudinale de la plante, montre qu'elle reste cantonnée dans l'espace intertidal entre le niveau atteint par les plus hautes mers et celui des pleines mers de mortes-eaux de coefficient 40 , soit entre $4,9 \mathrm{~m}$ et $7,5 \mathrm{~m}$ au-dessus du zéro hydrographique dans un contexte macrotidal où le marnage atteint $7,32 \mathrm{~m}$. Ceci correspond à une fréquence de submersion par la mer comprise entre 0 et $27 \%$. Ces valeurs sont comparables à celles qu'indiquent P. Cranford et al., (1989), dans la baie de Fundy, où avec un marnage de 12 mètres, la limite basse de l'espèce se situe à 2 mètres sous le niveau des pleines mers moyennes. Par contre, elles s'écartent sensiblement de celles qu'ont relevées T. McKee et W. Patrick (1988) dans les marais de la côte est des États-Unis, où en contexte micro à mésotidal, Spartina alterniflora est présente sur tout l'estran. La hauteur d'eau et le temps de submersion apparaissent donc comme des facteurs déterminants pour la répartition altitudinale de l'espèce. L'éradication de la Spartine en rade de Brest, dans le but de protéger le Limonium, apparaitt toutefois difficile à mettre en œuvre du fait du contexte géographique local, qui s'oppose à la mise en œuvre de techniques lourdes ou à grande échelle. Un des modes de gestion du problème passe par des mesures souples de type «jardinage écologique », comme l'arrachage manuel, etc., là où les facteurs naturels limitent déjà l'expansion de la Spartine.

\section{BIBLIOGRAPHIE}

BONNOT-COURTOIS (C.), GUÉNÉGOU (M.-C.), LAFOND (L.-R.), LEVASSEUR (J.-E.), 1989. - Dynamique sédimentaire et colonisation végétale dans l'anse du Kernic (Finistère nord), Mémoire du Laboratoire de Géomorphologie, Dinard, EPHE, 42, $122 \mathrm{p}$.

BROOME (S.), SENECA (E.), WOODHOUSE (W.), 1986. - « Long-term growth and development of transplants of the salt-marh grass Spartina alterniflora », Estuaries, vol. 9, $n^{\circ} 1$, p. 63-74. 
CALLAWAY (J.), JOSSELYN (M.), 1992. - « The introduction and spread of smooth cordgrass (Spartina alterniflora) in south san francisco Bay », Estuaries, vol. 15, n² 2, p. 218-226.

CHUNG (C. H.), 1983. - « Geographical distribution of Spartina anglica C.E. Hubbard in China », Bulletin of Marine Science, $\mathrm{n}^{\circ}$ 33, p. 753-758.

CRANFORD (P.), GORDON (D.), JARVIS (C.), 1989. - « Measurement of cordgrass, spartina alterniflora, production in a macrotidal estuary, bay of Fundy ", Estuaries, vol. 12, n 1, p. 27-34.

DAEHLER (C.), 1996. - « Spartina invasions in Pacific estauries : biology, impact, and managment », Proceedings of Symposium on non-indigenous Species in Western Aquatic Ecosystems, Portland State Univ. Lakes and Reservoirs Prog. 96, Systema editor, 8 p.

DAEHLER (C.), STRONG (D.), 1996. - « Status, prediction and prevention of introduced cordgrass Spartina spp. Invasions in Pacific estuaries, USA », Biological Conservation, vol. 78, p. 51-58.

DAVEY (P.), 1993. - «Spartina : eliminating the root of the problem », Enact, Managing land for the wildlife, vol. $1, \mathrm{n}^{\circ} 3, \mathrm{p} .7$.

FEIST (B. E.), SIMENSTAD (C. A.), 2000. - « Expansion rates and recruitment frequency of exotic smooth cordgrass, Spartina alterniflora (Loisel), colonizing unvegetated littoral flats in Willapa bay, Washington ", Estuaries, vol. 23, n² 2, p. 267-274.

FiCHAUT (B.), SPARFEL (L.), SUANEZ (S.), 2004. - Rapport d'étude sur la progression de la Spartine (Spartina alterniflora) et sur la dynamique sédimentaire en rade de Brest. Sites de Pont-Callec, du pédel et de Mengleuz, Géomer - LETG UMR 6554 CNRS, IUEN (Plouzané), décembre 2004, 49 p.

GARNETT (R. P.), HIRONS (G.), EVANS (C.), O'CONNOR (D.), 1992. - « The control os Spartina (cord-grass) using glyphosate ", Aspects of Applied Biology, vol. 29, p. 359-364.

GREVSTAD (F. S.), STRONG (D. R.), GARCIA-ROSSI (D.), SWITZER (R. W.), WECKER (M. S.), 2003. - « Biological control of Spartina alterniflora in Willapa Bay, Washington using the planthopper Prokelisia marginata : agent specificity and early results ", Biological Control, $n^{\circ} 27$, p. 32-42.

GROSS (M.), KLEMAS (V.), LEVASSEUR (J.-E.), 1986. - « Biomass and structure of a Spartina alterniflora dominated salt-marsh in France », Bulletin of the Torrey Botanical Club, $\mathrm{n}^{\circ} 113$, p. 125-130.

GUÉNÉGOU (M.-C.), LEVASSEUR (J.-E.), BONNOT-COURTOIS (C.), LAFOND (L.-R.), LE RHUN (J.), 1991. - « The geomorphological and botanical changes in Kernic bay (Brittany, France) : influence on coastal management », Journal of Coastal Research, vol. 7, n 2, p. 331-339.

GUILCHER (A.), ANDRADE (B.), DANTEC (M.-H.), 1982. - « Diversité morpho-sédimentologique des estuaires du Finistère ", Norois, $n^{\circ}$ 114, 205-228.

HUBBARD (C. E.), 1992. - Grasses. A guide to their Structure, Identification, Uses, and Distribution in the British Isles, London, Penguin Books, $4^{\mathrm{e}}$ édition, $476 \mathrm{p}$.

LEVASSEUR (J.), GUENEGOU (M.-C.), 1988. - «Évaluation du rôle joué par les plantes supérieures dans le comblement de la Baie du Kernic (Finistère), cas de Spartina anglica C. E. Hubbard ", Rapport final de contrat $n^{\circ} K C 2001$, Université de Rennes I, 28 p.

LINTHURST (R. A.), SENECA (E. D.), 1981. - « Aeration, nitrogen and salinity as determinants of Spartina alterniflora Loisel. Growth response ", Estuaries, vol. 4, n 1, p 53-63.

MACKEE (K.), PATRICK (W.), 1988. - « The relationship of smooth cordgrass (Spartina alterniflora) to tidal datums : a review ", Estuaries, vol. 11, n 3, p. 143-151. 
MARCHANT (C. J.), 1968a. - « Evolution in Spartina(Gramineae). II. Chromosomes, basic relationships and the problem of S. $x$ townsendii agg. ", Journal of the Linnean Society (Botany), 60, p. 381-409.

MARCHANT (C. J.), 1968b. - « Evolution in Spartina (Gramineae). III. Species chromosomes number and their taxonomic significance », Journal of the Linnean Society (Botany), 60, p. 411-417.

QUÉRÉ (E.), MAGNANON (S.), 1998. - Étude des populations de Limonium humile en rade de Brest - bilan des trois années de suivi, proposition de mesures de gestion, Conservatoire Botanique National de Brest, Rapport pour la CUB - Cellule Rade, non publié, 18 p.

-, 2000. - La sauvegarde des populations de Limonium humile en rade de Brest, Conservatoire Botanique National de Brest, Rapport pour la CUB, non publié, 14 p.

REIDENBAUGH (T.), 1983. - « Productivity of cordgrass, Spartina alterniflora, estimated from live standing crops, mortality, and leaf shedding in a Virginia salt marsh », Estuaries, vol. 6, $\mathrm{n}^{\circ} 1, \mathrm{p}$. 57-65.

SPARFEL (L.), 2004. - Progression de la Spartine (Spartina alterniflora) et dynamique sédimentaire en rade de Brest (Finistère) : de la mesure à la réponse gestionnaire. Ces des sites de Pont-Callec, le Pédel et Mengleuz, Mémoire de Maîtrise, Université de Bretagne Occidentale, 217 p.

SOURNiA (A.), FAGOT (C.), TRIPLET (P.), DESPREZ (M.), 2000. - Contrôle de la Spartine en baie de Somme : contribution à la réflexion, Syndicat mixte pour l'aménagement de la côte picarde/GEMEL Picardie/ RAMSAR/Réserve naturelle de la baie de Somme, $89 \mathrm{p}$.

SWALES (A.), MACDONALD (I. T.), GREEN (M. O.), 2004. - « Influence of wave and sediment dynamics on cordgrass (Spartina anglica) growth and sediment accumulation on an exposed intertidal flat ", Estuaries, vol. 27, $\mathrm{n}^{\circ}$ 2, p. 225-243.

TESSON (Y.), QUERE (E.), MAGNANON (S.), 1997. - Suivi des populations de Limonium humile en rade de Brest, Conservatoire Botanique National de Brest, Rapport pour la CUB - Cellule Rade, non publié, 9 p. VERGER (F.), 2005. - Marais maritimes et estuaires du littoral français, Paris, Belin, 335 p.

\section{NOTES}

1. L'appellation Brest Métropole Océane (BMO) remplace le sigle CUB (Communauté Urbaine de Brest) depuis le mois de janvier 2005.

2. Le type d'appareil utilisé correspond à une station Trimble 5700/5800.

3. La borne en granite IGN située au nord du hameau de Rosmélec (commune de Daoulas) porte le $\mathrm{n}^{\circ}$ de site 2904301 (altitude IGN69: 54,69 m) ; celle située au sud du port du Tinduff (commune de Plougastel-Daoulas) porte le numéro de site 2918907 (altitude IGN69 : 28,39 m).

\section{RÉSUMÉS}

L'étude de la progression de la Spartine (Spartina alterniflora Loisel) en rade de Brest entre 1952 et 2004 a été réalisée à partir d'une analyse diachronique par photo-interprétation et de mesures de terrain au DGPS. Les résultats montrent des vitesses de colonisation importantes des hautes 
slikkes donnant des taux d'expansion annuelle équivalents compris entre $4 \%$ et $24 \%$ suivant les sites. Les modalités de son installation et de sa propagation varient d'un site à l'autre en fonction de facteurs écologiques limitants tels que l'hydrodynamisme et ses conséquences sur la nature du substrat ou le temps de submersion par les eaux marines. L'analyse de la répartition altitudinale de la plante montre qu'elle peut coloniser tout l'espace littoral compris entre le niveau atteint par les plus hautes mers et celui des pleines mers de mortes eaux de coefficient 40 . Des mesures sont proposées dans le but de stopper localement sa progression qui se fait, entre autres, aux dépens d'une espèce endémique à la rade de Brest, le Limonium humile (Miller). Elles consistent en la mise en œuvre d'interventions très souples de type arrachage manuel ou utilisation de barrière PVC.

The study of spread of smooth cordgrass - Spartina alterniflora (Loisel) - in the rade of Brest between 1952 and 2004 was realised using aerial photographs diachronic analysis and DGPS field measurements. The results showed that propagation speeds were important inducing annual expansion rates reaching $4 \%$ to $24 \%$ depending on sites. The introduction and spread patterns differ from one site to the other depending on limiting ecological factors such as hydrodynamic conditions and its implications on substratum characteristics or time submersion by marine water. The analysis of vertical distribution of the plant shows that it is able to colonize all the upper intertidal zone beetween the highest spring tide level and the high neap tide level. Methods are proposed to locally prevent its progression that occurs at the expense of Limonium humile (Miller), an endemic species of the rade of Brest. They consist in soft interventions as manual uprooting or burying of vertical plastic sheets around the spartina spots.

\section{INDEX}

Keywords : aerial photograph analysis, expansion, management

Mots-clés : DGPS, limonium, photo-interprétation, progression

Index géographique : France, Brest, Brest (rade de)

\section{AUTEURS}

\section{LENAIIG SPARFEL}

Géomer - UMR 6554 CNRS LETG, Université de Bretagne Occidentale, IUEM, Technopôle BrestIroise, Place Nicolas Copernic, 29280 Plouzané, France, lenaig.sparfel@univ-brest.fr

\section{BERNARD FICHAUT}

Géomer - UMR 6554 CNRS LETG, Université de Bretagne Occidentale, IUEM, Technopôle BrestIroise, Place Nicolas Copernic, 29280 Plouzané, France, bernard.fichaut@univ-brest.fr

\section{SERGE SUANEZ}

Géomer - UMR 6554 CNRS LETG, Université de Bretagne Occidentale, IUEM, Technopôle BrestIroise, Place Nicolas Copernic, 29280 Plouzané, France, serge.suanez@univ-brest.fr 